\title{
A Rare Cause of Palpitations
}

\section{Gonzalez L ${ }^{1^{*}}$ and Andy Beale ${ }^{2}$}

${ }^{1}$ Acute Physician in AAU, Great Western Hospital, Swindon, United Kingdom

${ }^{2}$ Consultant Radiologist, Great Western Hospital, Swindon, United Kingdom

*Corresponding author: Gonzalez L, Acute Physician in AAU, Great Western Hospital, Swindon, UK, E-mail: lauravgonzalez@googlemail.com

Citation: Gonzalez L, Andy Beale (2017) A Rare Cause of Palpitations. SAJ Case Reports 4: 201

Article history: Received: 23 February 2017, Accepted: 20 March 2017, Published: 22 March 2017

\section{Introduction}

An 87 years lady presented to the Acute Medical Unit with palpitations and breathlessness. She was haemodinamically stable and ECG was normal in sinus rhythm but cardiac monitoring demonstrated runs of atrial fibrillation. Bloods including TSH were normal. CxR (Figure 1) demonstrated opacity in Right Lower Lobe. A CT scan (Figure 2 and Figure 3) and Echocardiogram (Figure 4) was undertaken.

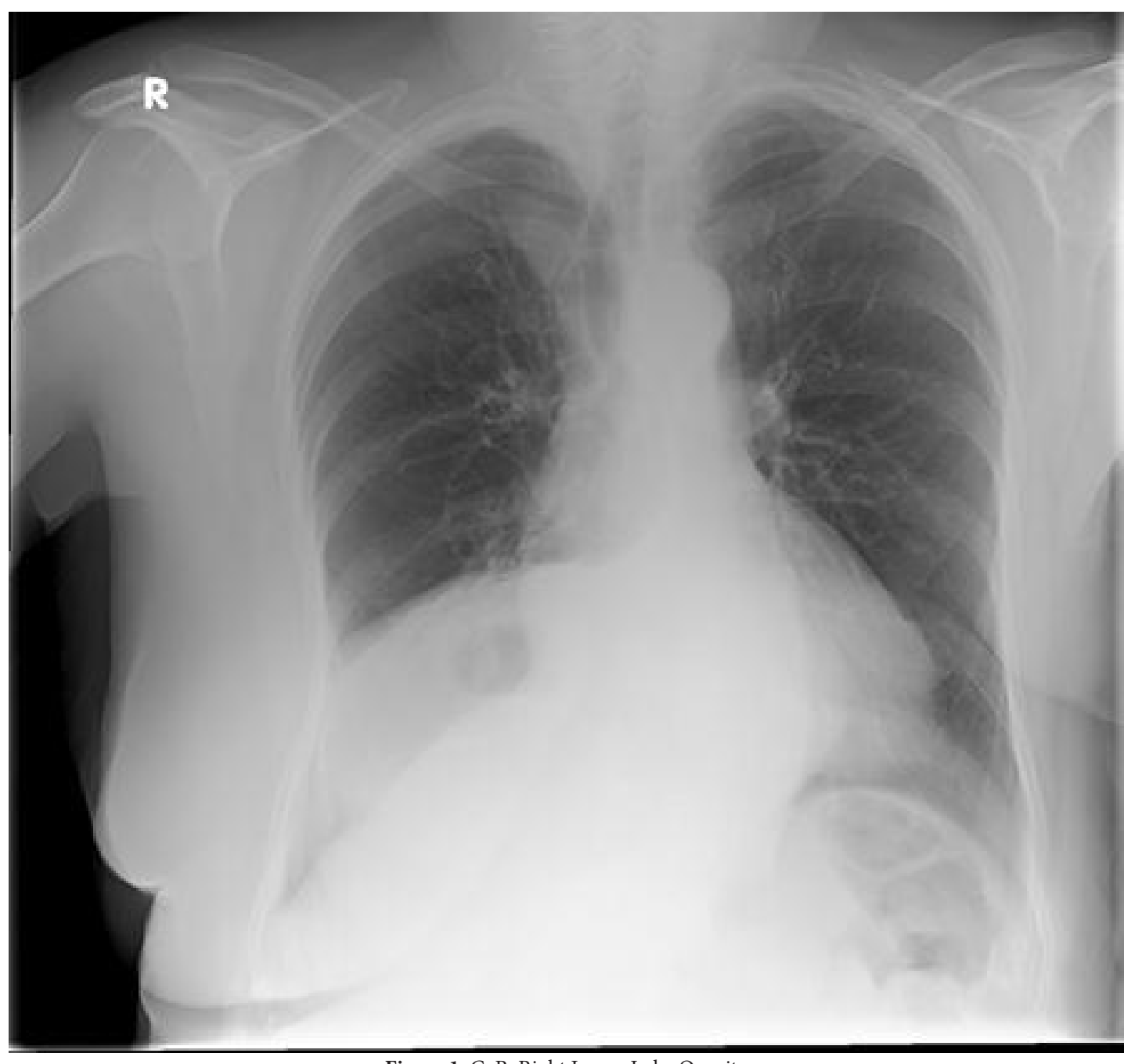

Figure 1: CxR: Right Lower Lobe Opacity 
The CT scan showed a large posterior diaphragmatic hernia containing spleen, stomach and tail of pancreas.

Common cause of diaphragmatic hernias in adults are trauma, congenital defect in the diaphragm, iatrogenic injury during abdominal or thoracic surgery and factors increasing intra-abdominal pressure such as pregnancy, child birth, coughing and sneezing.

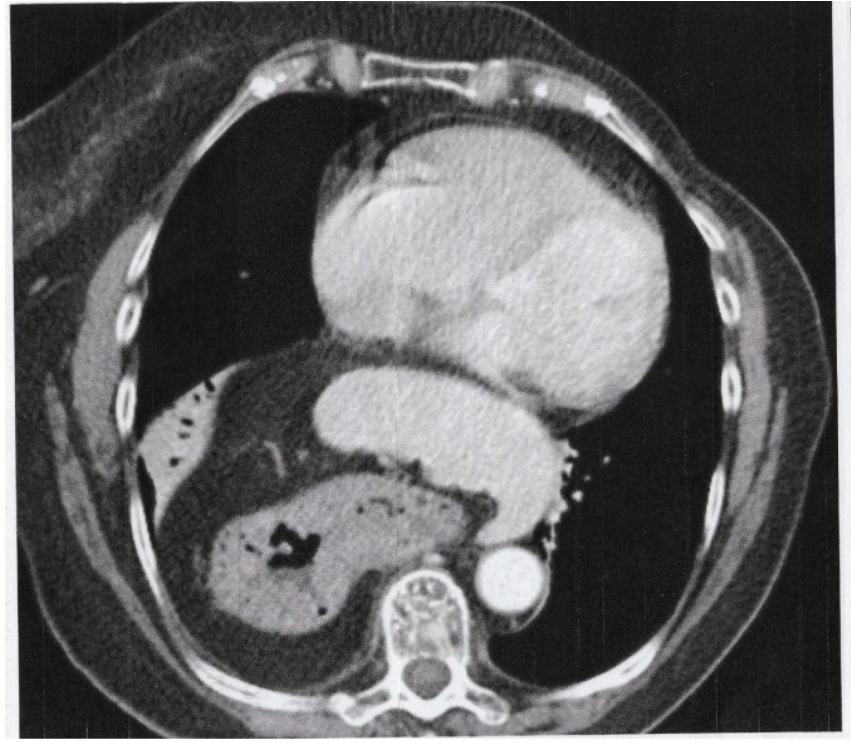

Figure 2

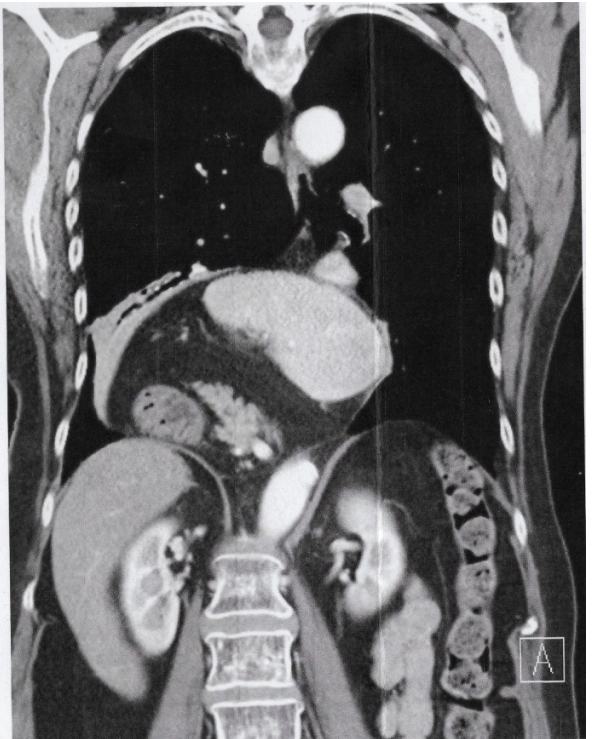

Figure 3

The majority of Bochdalek hernias are asymptomatic and are usually incidental findings. Symptoms are depending on the defect size and hernia contents and can be pulmonary, gastrointestinal and rarely cardiac.

The major complication is strangulation of the herniated contents, as this is associated with mortality of over $80 \%$. This represents a surgical emergency and requires prompt diagnosis and surgical repair.

Our case could be managed with symptoms control with Bblockers or Diltiazem.

Surgery is required in strangulation and very symptomatic hernias, small defects are easier to repair whereas larger defects may involve reduction of intraabdominal contents.

Echocardiogram showed a large extra cardiac structured below the left atrium which was the spleen.

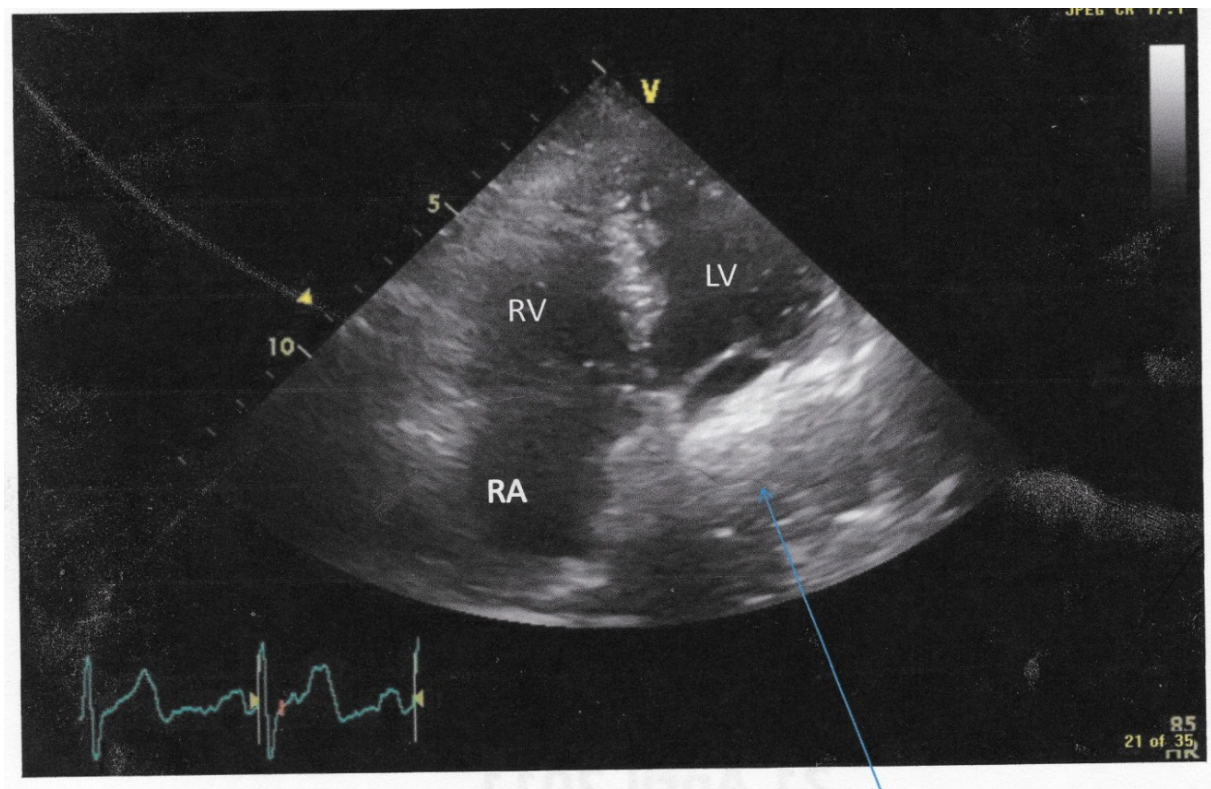

Mass is compressing in on the LA so you can barley see the LA.

Figure 4: Echocardiogram showed a large extra cardiac structured below the left atrium which was the spleen. 


\section{References}

1. Aydın Kurt, KR Yazıcıoğlu, A İpek, ÖTosun, M Coşkun (2004) Right sided diaphragmatic hernia in an adult without history of trauma. Unusual CT findings. Eur J Gen Med 1: 55-7.

2. Shin MS, Mulligan SA, Baxley WA, Ho KJ (1987) Bochdalek Hernia of Diaphrragm in the Adult: Diagnosis by Computed Tomography. Chest 92: 1098-101.

3. Gale ME (1985) Bochdalek Hernia: Prevalence and CT Characteristics. Radiology 156: 449-52. 